\title{
THE CLOSURE OF A LOCAL SUBEXPONENTIAL DISTRIBUTION CLASS UNDER CONVOLUTION ROOTS, WITH APPLICATIONS TO THE COMPOUND POISSON PROCESS
}

\author{
YUEBAO WANG, ${ }^{* * *}$ \\ DONGYA CHENG ${ }^{*}$ AND \\ KAIYONG WANG, ${ }^{*}$ Soochow University
}

\begin{abstract}
Let $s_{\Delta}$ denote the class of local subexponential distributions and $F^{* \nu}$ the $v$-fold convolution of distribution $F$, where $v$ belongs to one of the following three cases: $v$ is a random variable taking only a finite number of values, in particular $v \equiv n$ for some $n \geq 2$; $v$ is a Poisson random variable; or $v$ is a geometric random variable. Along the lines of Embrechts, Goldie, and Veraverbeke (1979), the following assertion is proved under certain conditions: $F^{* \nu} \in s_{\Delta} \Longleftrightarrow F \in s_{\Delta}$. This result is applied to the infinitely divisible laws and some new results are established. The results obtained extend the corresponding findings of Asmussen, Foss, and Korshunov (2003).
\end{abstract}

Keywords: Local subexponential distribution class; convolution root; compound Poisson process; infinitely divisible law

2000 Mathematics Subject Classification: Primary 60E05

Secondary 60F99

\section{Introduction}

Recently, Asmussen et al. (2003) introduced the notion of a local subexponential distribution class and investigated its properties systematically. They applied the obtained properties to risk theory, the compound Poisson process, the compound geometric process, infinitely divisible laws, and branching processes. They also obtained a series of important results, which included two equivalent conditions for the local asymptotic behavior of the compound Poisson process.

The motivation of the present paper comes from the local asymptotic behavior of the compound Poisson process. Since the compound Poisson process is a special process with good properties, it is reasonable to believe that its local asymptotic behavior has richer contents then some general processes, i.e. more equivalent conditions. In fact, the nonlocal asymptotic behavior of the compound Poisson process had three equivalent conditions (see Theorem 3 of Embrechts et al. (1979)), which are richer than the local asymptotic behavior (see Theorem 6 of Asmussen et al. (2003)). In Section 3, we will establish a local version of Theorem 3 of Embrechts et al. (1979). Since the compound geometric process can be transformed to the

Received 5 April 2005; revision received 2 July 2005.

* Postal address: Department of Mathematics, Soochow University, Suzhou, 215006, P. R. China.

** Email address: ybwang@suda.edu.cn 
compound Poisson process, we will also present three equivalent conditions for local asymptotic behavior of the compound geometric process in Section 3. In Section 4, we will apply the result on the compound Poisson process to infinitely divisible laws and discuss the relation between an infinitely divisible local subexponential distribution and its Lévy measure. The obtained results extend the corresponding results of Asmussen et al. (2003).

To realize the above goals, we first need solve a crucial problem, as did Embrechts et al. (1979), that is, proving the closure of a local subexponential distribution class under convolution roots. We will discuss this explicitly in Section 2.

Before doing this, we introduce some notions, notation, and conventions, which will be valid in the rest of this paper. We assume that the support of the distribution $F$ is $[0, \infty)$. If $F$ is proper, i.e. $F(\infty)=1$, we write $\bar{F}(x)=1-F(x), x \in(-\infty, \infty)$. If $F$ is not proper, i.e. $F(\infty)<\infty$ but $F(\infty) \neq 1$, we write $F_{0}(x)=F(x)(F(\infty))^{-1}, x \in(-\infty, \infty)$, i.e. $F_{0}$ is a proper distribution. We denote by $F^{* n}$ the $n$-fold convolution of distribution $F$, for some $n \geq 2$. Let $0<T \leq \infty$ and $\Delta=(0, T]$; when $T=\infty, \Delta$ reduces to $(0, \infty)$. Write $x+\Delta=\{x+y, y \in \Delta\}, x \in(-\infty, \infty)$. Unless stated otherwise, all limiting relationships are for $x \rightarrow \infty$, and we write $a(x) \sim b(x)$ if $a(x) / b(x) \rightarrow 1$. If there exists some $b, 0<b<\infty$ such that $\limsup _{x \rightarrow \infty} a(x)(b(x))^{-1} \leq b$, then we write $a(x)=O(1) b(x)$. If $a(x)=O(1) b(x)$ and $b(x)=O(1) a(x)$, then we write $a(x) \approx b(x)$.

Asmussen et al. (2003) introduced some important local distribution classes.

We say that a distribution belongs to the class $\mathcal{L}_{\Delta}$ if, for all sufficiently large $x, F(x+\Delta)>0$ and $F(x+y+\Delta) \sim F(x+\Delta)$ uniformly in $|y| \leq 1$. We say that $F$ belongs to the local subexponential distribution class $\varsigma_{\Delta}$ if $F \in \mathcal{L}_{\Delta}$ and $F_{0}^{* 2}(x+\Delta) \sim 2 F_{0}(x+\Delta)$.

Let

$$
\begin{aligned}
\mathscr{H}_{\Delta}(F)= & \left\{h:[0, \infty) \rightarrow[0, \infty): h(x) \uparrow \infty, x^{-1} h(x) \rightarrow 0,\right. \\
& F(x+y+\Delta) \sim F(x+\Delta) \text { uniformly in } y,|y| \leq h(x)\} .
\end{aligned}
$$

Asmussen et al. (2003) contained the following simple but useful fact:

$$
F \in \mathcal{L}_{\Delta} \Longleftrightarrow \mathscr{H}_{\Delta}(F) \neq \varnothing .
$$

Now we return to the closure of a local subexponential distribution class under convolution roots. The question is: under which conditions imposed on $F$ can $F^{* n} \in s_{\Delta} \Longleftrightarrow F \in s_{\Delta}$ ? Furthermore, let $\left\{X_{k}, k \geq 1\right\}$ be a sequence of independent and identically distributed (i.i.d.) random variables (RVs) with common distribution $F$ on $[0, \infty)$. Write $F^{* v}(x)=\mathrm{P}\left(S_{v} \leq x\right)$, $x \in(-\infty, \infty)$, where $S_{v}=\sum_{k=1}^{v} X_{k}, S_{0}=0$, and $v$ is a nonnegative integer RV and is independent of $\left\{X_{k}, k \geq 1\right\}$. Naturally, we should exclude the case that $v \equiv 0$, so we always assume that $\mathrm{P}(v \geq 1)>0$. The more general question is: under which conditions imposed on $\nu$ and $F$ can $F^{* \nu} \in s_{\Delta} \Longleftrightarrow F \in s_{\Delta}$ ? This paper provides some positive answers to this question in the following cases:

(i) $v$ is an RV taking only a finite number of values, in particular $v \equiv n$ for some $n \geq 2$,

(ii) $v$ is a Poisson RV,

(iii) $v$ is a geometric RV.

For any RV $v$ with a light-tailed distribution or, more generally, for any RV $v$, it is unknown whether or not

$$
F^{* \nu} \in s_{\Delta} \Longleftrightarrow F \in \varsigma_{\Delta} \text {. }
$$




\section{Results in case (i)}

In this section, we assume that the distribution $F$ is proper and that $0<T<\infty$. We first discuss a particular case, that is $v \equiv n$ for some $n \geq 2$. We need the following lemma.

Lemma 2.1. Let $F^{* v} \in \mathcal{L}_{\Delta}$, and let $F \in \mathcal{L}_{\Delta}$ or $\mathrm{P}(v=1)=p_{1}>0$. Then, for any positive integer $m \geq 1$,

$$
F^{*(v+m)} \in s_{\Delta} \Longleftrightarrow F^{* v} \in s_{\Delta},
$$

and either $F^{*(\nu+m)} \in \varsigma_{\Delta}$ or $F^{* \nu} \in \varsigma_{\Delta}$ implies that

$$
F^{*(v+m)}(x+\Delta) \approx F^{* v}(x+\Delta)
$$

Proof. By induction, we only need to prove the case $m=1$. Take any $h \in \mathscr{H}_{\Delta}\left(F^{* \nu}\right)$. Then we obtain

$$
\begin{aligned}
F^{*(v+1)}(x+\Delta)= & \left(\int_{0}^{h(x)}+\int_{h(x)}^{x-h(x)}+\int_{x-h(x)}^{x}\right) F^{* v}(x-y+\Delta) F(\mathrm{~d} y) \\
& +\int_{x}^{x+T} F^{* v}(x-y+T) F(\mathrm{~d} y) \\
= & : \sum_{k=1}^{4} I_{k}(x) .
\end{aligned}
$$

Firstly, we prove that $F^{* \nu} \in \varsigma_{\Delta} \Rightarrow F^{*(\nu+1)} \in \varsigma_{\Delta}$. By $F^{* \nu} \in \mathcal{L}_{\Delta}$, we know that

$$
I_{1}(x) \sim F^{* v}(x+\Delta)=O(1) F^{*(v+1)}(x+\Delta) .
$$

When $F \in \mathcal{L}_{\Delta}$, recall that $\mathrm{P}(v \geq 1)>0$. Then, by Fatou's lemma and Corollary 1 of Asmussen et al. (2003), we have

$$
\begin{aligned}
\liminf _{x \rightarrow \infty} F^{* v}(x+\Delta)(F(x+\Delta))^{-1} & \geq \liminf _{x \rightarrow \infty} \sum_{n=1}^{\infty} F^{* n}(x+\Delta)(F(x+\Delta))^{-1} \mathrm{P}(v=n) \\
& \geq \sum_{n=1}^{\infty} \liminf _{x \rightarrow \infty} F^{* n}(x+\Delta)(F(x+\Delta))^{-1} \mathrm{P}(v=n) \\
& \geq \sum_{n=1}^{\infty} n \mathrm{P}(v=n) \\
& \geq \mathrm{P}(v \geq 1) \\
& >0 .
\end{aligned}
$$

Hence,

$$
F(x+\Delta)=O(1) F^{* v}(x+\Delta) .
$$


If $p_{1}>0$, then (2.2) clearly holds. Using integration by parts, (2.2), $F^{* v} \in s_{\Delta}$, and Proposition 2 of Asmussen et al. (2003), we have

$$
\begin{aligned}
I_{2}(x)= & \int_{h(x)}^{x-h(x)}\left(\overline{F^{* v}}(x-y)-\overline{F^{* \nu}}(x-y+T)\right) F(\mathrm{~d} y) \\
= & \int_{h(x)}^{x-h(x)} \bar{F}(x-y) \overline{F^{* \nu}}(\mathrm{d} y)-\int_{h(x)+T}^{x-h(x)+T} \bar{F}(x-y+T) \overline{F^{*}}(\mathrm{~d} y) \\
& -\bar{F}(x-h(x)) \overline{F^{* \nu}}(h(x)+\Delta)+\bar{F}(h(x)) \overline{F^{* \nu}}(x-h(x)+\Delta) \\
= & \int_{h(x)}^{x-h(x)} F(x-y+\Delta) F^{* v}(\mathrm{~d} y)+\int_{h(x)}^{h(x)+T} \bar{F}(x-y+T) F^{*}(\mathrm{~d} y) \\
& -\int_{x-h(x)}^{x-h(x)+T} \bar{F}(x-y+T) \overline{F^{*}}(\mathrm{~d} y) \\
& -\bar{F}(x-h(x)) \overline{F^{* \nu}}(h(x)+\Delta)+\bar{F}(h(x)) \overline{F^{* \nu}}(x-h(x)+\Delta) \\
\leq & \int_{h(x)}^{x-h(x)+T} F(x-y+\Delta) F^{* \nu}(\mathrm{d} y) \\
= & O(1) \int_{h(x)}^{x-h(x)+T} F^{* \nu}(x-y+\Delta) F^{* \nu}(\mathrm{d} y) \\
= & o(1) F^{* \nu}(x+\Delta) .
\end{aligned}
$$

Using integration by parts again, we obtain

$$
\begin{aligned}
I_{3}(x)= & -\bar{F}(x) F^{* v}(T)+\bar{F}(x-h(x)) F^{* \nu}(h(x)+\Delta) \\
& +\int_{0}^{h(x)+T} F(x-y+\Delta) F^{* v}(\mathrm{~d} y)-\int_{h(x)}^{h(x)+T} \bar{F}(x-y) F^{* v}(\mathrm{~d} y) \\
& +\int_{0}^{T} \bar{F}(x-y+T) F^{* \nu}(\mathrm{d} y)
\end{aligned}
$$

and

$$
I_{4}(x)=\bar{F}(x) F^{* \nu}(T)-\int_{0}^{T} \bar{F}(x-y+T) F^{* \nu}(\mathrm{d} y)
$$

Write

$$
I_{5}(x)=\int_{h(x)}^{h(x)+T} \bar{F}(x-y) F^{* v}(\mathrm{~d} y)-\bar{F}(x-h(x)) F^{* \nu}(h(x)+\Delta) .
$$

Then, by (2.2) and $F^{* v} \in \mathcal{L}_{\Delta}$, we have

$$
\begin{aligned}
0 & \leq I_{5}(x) \\
& \leq F(x-h(x)-T+\Delta) F^{* v}(h(x)+\Delta) \\
& =o(1) F^{* v}(x+\Delta) .
\end{aligned}
$$

By (2.2) and (2.4)-(2.6), we know that

$$
I_{3}(x)+I_{4}(x)=O(1) F^{* v}(x+\Delta) .
$$


By (2.1), (2.3), and (2.7), we know that $F^{*(v+1)}(x+\Delta) \approx F^{* v}(x+\Delta)$. By (2.1), (2.3), (2.6), and $F \in \mathcal{L}_{\Delta}$, we know that

$$
\begin{aligned}
F^{*(v+1)}(x+\Delta) & \sim F^{* v}(x+\Delta)+\int_{0}^{h(x)+T} F(x-y+\Delta) F^{* v}(\mathrm{~d} y) \\
& \sim F^{* v}(x+\Delta)+F(x+\Delta),
\end{aligned}
$$

so that $F^{*(v+1)} \in \mathcal{L}_{\Delta}$. Hence, by Lemma 1 of Asmussen et al. (2003) and $F^{* v} \in \varsigma_{\Delta}$, $F^{*(v+1)} \in \varsigma_{\Delta}$ follows.

Next, we prove that $F^{*(v+1)} \in \varsigma_{\Delta} \Rightarrow F^{* v} \in s_{\Delta}$. By $F^{* v} \in \mathcal{L}_{\Delta}$ and $F \in \mathcal{L}_{\Delta}$ or $p_{1}>0$, we know that (2.1), (2.2), and (2.7) still hold. By virtue of (2.1), integration by parts, (2.2), $F^{*(v+1)} \in s_{\Delta}$, and Proposition 2 of Asmussen et al. (2003), it follows that

$$
\begin{aligned}
I_{2}(x) & =O(1) \int_{h(x)}^{x-h(x)} F^{*(v+1)}(x-y+\Delta) F(\mathrm{~d} y) \\
& =O(1) \int_{h(x)}^{x-h(x)+T} F(x-y+\Delta) F^{*(v+1)}(\mathrm{d} y) \\
& =O(1) \int_{h(x)}^{x-h(x)+T} F^{*(v+1)}(x-y+\Delta) F^{*(v+1)}(\mathrm{d} y) \\
& =o\left(F^{*(v+1)}(x+\Delta)\right) .
\end{aligned}
$$

By (2.1), (2.7), and (2.8), we know that $F^{*(v+1)}(x+\Delta) \approx F^{* v}(x+\Delta)$. Hence, by $F^{* v} \in \mathcal{L}_{\Delta}$, we have $F^{* \nu} \in s_{\Delta}$.

Corollary 2.1. If $F \in \mathcal{L}_{\Delta}$ then, for any positive integer $n \geq 2, F^{* n} \in s_{\Delta} \Longleftrightarrow F \in s_{\Delta}$, and either $F^{* n} \in s_{\Delta}$ or $F \in s_{\Delta}$ implies that $F^{* n}(x+\Delta) \approx F(x+\Delta)$.

Proof. The result follows by substituting $v=1$ and $m=n-1$ into Lemma 2.1.

The result in case (i) is implied by the following lemma.

Lemma 2.2. If $F \in \mathcal{L}_{\Delta}, F^{* v} \in \mathcal{L}_{\Delta}$, and the distribution of $v$ is $\mathrm{P}(v=i)=p_{i}, i=$ $1,2, \ldots, s$, then, for any positive integer $m \geq s+1, F^{* m} \in s_{\Delta} \Longleftrightarrow F^{* \nu} \in s_{\Delta}$, and either $F^{* m} \in s_{\Delta}$ or $F^{* v} \in s_{\Delta}$ implies that $F^{*(v+m)}(x+\Delta) \approx F^{* v}(x+\Delta)$.

Proof. By Lemma 2.1, we only need to prove the case $m=s+1$. By $F \in \mathcal{L}_{\Delta}$ and Proposition 1 of Asmussen et al. (2003), we get $F^{* i} \in \mathcal{L}_{\Delta}$ for all $i \geq 1$. By $p_{s}>0$ and

$$
F^{* v}(x+\Delta)=\sum_{i=1}^{s} p_{i} F^{* i}(x+\Delta)
$$

we know that $F^{* s}(x+\Delta)=O(1) F^{* v}(x+\Delta)$. By $F \in \mathcal{L}_{\Delta}$, we have $F^{* i} \in \mathcal{L}_{\Delta}, i=$ $2,3, \ldots, s$. Hence, there exists an $h \in \bigcap_{i=2}^{s-1} \mathscr{H}_{\Delta}\left(F^{* i}\right)$ such that

$$
F^{* s}(x+\Delta) \geq \int_{0}^{h(x)} F^{* i}(x-y+\Delta) F^{*(s-i)}(\mathrm{d} y) \sim F^{* i}(x+\Delta), \quad i=2, \ldots, s-1 .
$$

So

$$
\max \left\{F^{* i}(x+\Delta), i=1,2, \ldots, s\right\}=O(1) F^{* v}(x+\Delta) .
$$


Take any $h \in \bigcap_{i=1}^{s} \mathscr{H}_{\Delta}\left(F^{* i}\right) \cap \mathscr{H}_{\Delta}\left(F^{* v}\right)$. Then we obtain

$$
\begin{aligned}
F^{*(m+1)}(x+\Delta)= & \sum_{i=1}^{s}\left(p_{i}\left(\int_{0}^{h(x)}+\int_{h(x)}^{x-h(x)}+\int_{x-h(x)}^{x}\right) F^{* i}(x-y+\Delta) F^{*(m+1-i)}(\mathrm{d} y)\right. \\
& \left.\quad+\int_{x}^{x+T} F^{* i}(x-y+T) F^{*(m+1-i)}(\mathrm{d} y)\right) \\
= & : \sum_{k=1}^{4} I_{k}(x) .
\end{aligned}
$$

Firstly, we prove that $F^{* \nu} \in \varsigma_{\Delta} \Rightarrow F^{*(m+1)} \in \varsigma_{\Delta}$. By $F^{* i} \in \mathcal{L}_{\Delta}$, for all $i \geq 1$, and (2.10), we know that

$$
I_{1}(x) \sim \sum_{i=1}^{s} p_{i} F^{* i}(x+\Delta)=F^{* \nu}(x+\Delta)=O(1) F^{*(m+1)}(x+\Delta) .
$$

By (2.10), integration by parts, $F^{* \nu} \in \varsigma_{\Delta}$, Proposition 2 of Asmussen et al. (2003), and (2.11), we have

$$
\begin{aligned}
I_{2}(x) & =O(1) \sum_{i=1}^{s} p_{i} \int_{h(x)}^{x-h(x)} F^{* \nu}(x-y+\Delta) F^{*(m+1-i)}(\mathrm{d} y) \\
& =O(1) \sum_{i=1}^{s} p_{i} \int_{h(x)}^{x-h(x)+T} F^{*(m+1-i)}(x-y+\Delta) F^{* v}(\mathrm{~d} y) \\
& =O(1) \sum_{i=1}^{s} p_{i} \int_{h(x)}^{x-h(x)+T} F^{* v}(x-y+\Delta) F^{* v}(\mathrm{~d} y) \\
& =o\left(F^{* v}(x+\Delta)\right) .
\end{aligned}
$$

In a similar manner to (2.7), by (2.10) we know that

$$
I_{3}(x)+I_{4}(x) \sim \sum_{i=1}^{s} p_{i} F^{*(m+1-i)}(x+\Delta)=O(1) F^{* \nu}(x+\Delta) .
$$

By (2.11)-(2.13), we have $F^{*(m+1)}(x+\Delta) \approx F^{* \nu}(x+\Delta)$; hence $F^{*(m+1)} \in \wp_{\Delta}$ follows.

Next, we prove that $F^{*(m+1)} \in s_{\Delta} \Rightarrow F^{* \nu} \in s_{\Delta}$. By Corollary 2.1 and $F^{*(m+1)} \in s_{\Delta}$, we know that $F \in s_{\Delta}$; hence $F^{* \nu} \in s_{\Delta}$ follows from (2.9).

From the proof of Lemma 2.2, we immediately obtain the following theorem.

Theorem 2.1. With the conditions of Lemma 2.2, we obtain $F^{* \nu} \in s_{\Delta} \Longleftrightarrow F \in s_{\Delta}$, and either $F^{* v} \in s_{\Delta}$ or $F \in s_{\Delta}$ implies that $F^{*(v+m)}(x+\Delta) \approx F^{* v}(x+\Delta)$.

\section{Results in cases (ii) and (iii)}

In this section, we discuss results in cases (ii) and (iii), by applying Corollary 2.1 to the study of local asymptotic behavior for the compound Poisson process and the compound geometric process. 


\subsection{The compound Poisson process}

Let $F$ be a proper distribution. Assume that $v$ is the Poisson RV with parameter $\mu$ and let $G=F^{* \nu}$ be the compound Poisson distribution

$$
G(B)=\mathrm{e}^{-\mu} \sum_{n=0}^{\infty} \frac{\mu^{n}}{n !} F^{* n}(B) .
$$

It is well known that the compound Poisson process has many important applications in queueing, networks, finance and insurance, and other fields, so it has been extensively and deeply studied. Theorem 6 of Asmussen et al. (2003) described local asymptotic behavior for $G$.

Theorem 3.1. (Asmussen et al. (2003).) Assume that $T \leq \infty$. Furthermore, if $T<\infty$, then assume that $F \in \mathcal{L}_{\Delta}$. Then the following assertions are equivalent:

(i) $F \in s_{\Delta}$,

(ii) $G(x+\Delta) \sim \mu F(x+\Delta)$.

This result is a direct corollary of Theorem 2 of Asmussen et al. (2003). Just as it is pointed out in Section 1, the compound Poisson process is expected, as a special process, to have richer content than some general processes. As a matter of fact, when $T=\infty$, (i) and (ii) are also equivalent to $G \in \delta$ (see Embrechts et al. (1979, Theorem 3)). So, in the local version, with $T<\infty$, it is reasonable to expect that the corresponding equivalence holds. To demonstrate that it does so, define

$$
G^{* k^{-1}}(B) \equiv H(B)=\mathrm{e}^{-\mu k^{-1}} \sum_{n=0}^{\infty} \frac{\left(\mu k^{-1}\right)^{n}}{n !} F^{* n}(B), \quad \text { for all } k \geq 1 .
$$

Theorem 3.2. Let the conditions of Theorem 3.1 be valid. If there exists an integer $k=$ $k(\mu)>0$, such that $\mu k^{-1}<\ln 2$ and $G^{* k^{-1}} \in \mathcal{L}_{\Delta}$, then (i), (ii), and the following assertion are equivalent:

(iii) $G \in s_{\Delta}$.

We point out that our new conditions of Theorem 3.2 are similar to conditions used in Theorem 4.2 of Embrechts and Goldie (1982).

Proof of Theorem 3.2. We only need to prove the case $T<\infty$. Obviously, conditions (i) and (ii) imply condition (iii). Now, we follow the line of Embrechts et al. (1979) to prove that condition (iii) implies condition (i). We assume, in the first part of the proof, that $0<\mu<\ln 2$. Consider the proper distribution

$$
R(B)=\left(\mathrm{e}^{\mu}-1\right)^{-1} \sum_{n=1}^{\infty} \frac{\mu^{n}}{n !} G^{* n}(B)
$$

By the proof of Theorem 3 of Embrechts et al. (1979), we have $\mathrm{e}^{\mu} \bar{G}(x)=\left(\mathrm{e}^{\mu}-1\right) \bar{R}(x)$. Hence, for any $T, 0<T<\infty$,

$$
\mathrm{e}^{\mu} G(x+\Delta)=\left(\mathrm{e}^{\mu}-1\right) R(x+\Delta),
$$


giving $R \in s_{\Delta}$. Again by the proof of Theorem 3 of Embrechts et al. (1979), we know that $\mu \bar{F}(x)=-\sum_{n=1}^{\infty} n^{-1}\left(1-\mathrm{e}^{\mu}\right)^{n} \overline{R^{* n}}(x)$ and, hence, for any $T, 0<T<\infty$,

$$
\mu \frac{F(x+\Delta)}{R(x+\Delta)}=-\sum_{n=1}^{\infty} n^{-1}\left(1-\mathrm{e}^{\mu}\right)^{n} \frac{R^{* n}(x+\Delta)}{R(x+\Delta)} .
$$

Since $0<\mathrm{e}^{\mu}-1<1$, we can choose an $\varepsilon>0$ such that $\left(\mathrm{e}^{\mu}-1\right)(1+\varepsilon)<1$. Then, by $R \in s_{\Delta}$ and Proposition 4 of Asmussen et al. (2003), there exist an $x_{0}=x_{0}(\varepsilon)>0$ and a $V(\varepsilon)>0$ such that, for any $x \geq x_{0}$ and any $n \geq 1, R^{* n}(x+\Delta) / R(x+\Delta) \leq V(\varepsilon)(1+\varepsilon)^{n}$. Hence, using the dominated convergence theorem in (3.1), we obtain

$$
F(x+\Delta) \sim \mu^{-1}\left(1-\mathrm{e}^{-\mu}\right) R(x+\Delta),
$$

so $F \in \varsigma_{\Delta}$ follows.

Now consider an arbitrary $\mu>0$. Then there exists an integer $k=k(\mu)>0$ such that $\mu k^{-1}<\ln 2$ and $H \in \mathcal{L}_{\Delta}$. Again by the proof of Theorem 3 of Embrechts et al. (1979), we know that $H^{* k}=G \in s_{\Delta}$, giving $H \in s_{\Delta}$, by $H \in \mathcal{L}_{\Delta}$ and Corollary 2.1. The first part of the proof (since $\mu k^{-1}<\ln 2$ ) gives $F \in \wp_{\Delta}$.

\subsection{The compound geometric process}

Embrechts et al. (1979) pointed out that the compound geometric process could be rewritten as a compound Poisson process, so a similar result should also hold for the compound geometric process; we will state this without proof. Let $F$ be a proper distribution. Assume that $v$ is the geometric RV with parameter $\alpha$ and let $W=F^{* \nu}$ be the compound geometric distribution

$$
W(B)=(1-\alpha) \sum_{n=0}^{\infty} \alpha^{n} F^{* n}(B) .
$$

Theorem 3.3. Assume that $T \leq \infty$, and if $T<\infty$ then assume that $F \in \mathcal{L}_{\Delta}$. Then the following assertions are equivalent:

(i) $F \in s_{\Delta}$,

(ii) $W(x+\Delta) \sim \alpha(1-\alpha)^{-1} F(x+\Delta)$.

Furthermore, if there exists an integer $k=k(\mu)>0$ such that $2^{k}(1-\alpha)>1$ and $W^{* k^{-1}} \in \mathcal{L}_{\Delta}$, then (i) and (ii) are equivalent to the following assertion:

(iii) $W \in s_{\Delta}$.

We should point out that, as in Theorem 3.2, the only new result in Theorem 3.3 is that assertion (iii) implies assertion (i).

\section{Applications to infinitely divisible laws}

In this section, we will apply Theorem 3.2 to infinitely divisible laws to investigate the local relations between the infinitely divisible subexponential law and the corresponding Lévy measure.

The Laplace transform of an infinitely divisible law $F$ can be expressed as

$$
\int_{0}^{\infty} \mathrm{e}^{-\lambda x} F(\mathrm{~d} x)=\exp \left(-a \lambda-\int_{0}^{\infty}\left(1-\mathrm{e}^{-\lambda x}\right) v(\mathrm{~d} x)\right) .
$$


Here, $a \geq 0$ is a constant and the Lévy measure $v$ is a Borel measure on $(0, \infty)$ with the properties $\mu=v((1, \infty))<\infty$ and $\int_{0}^{1} x v(\mathrm{~d} x)<\infty$. Put $G(B)=v(B \cap(0, \infty)) \mu^{-1}$ and

$$
F_{2}(B)=\mathrm{e}^{-\mu} \sum_{n=0}^{\infty} \frac{\mu^{n}}{n !} G^{* n}(B)
$$

then $G$ and $F_{2}$ are proper distributions, and $F=F_{1} * F_{2}$, where $\overline{F_{1}}(x)=o\left(\mathrm{e}^{-\varepsilon x}\right)$ for every $\varepsilon>0$ (see Feller (1971)).

Theorem 7 of Asmussen et al. (2003) discussed the relation between $v(x+\Delta)$ and $F(x+\Delta)$.

Theorem 4.1. (Asmussen et al. (2003).) Assume that $T \leq \infty$. Furthermore, if $T<\infty$ then assume that $G \in \mathcal{L}_{\Delta}$. Then the following assertions are equivalent:

(i) $G \in s_{\Delta}$,

(ii) $v(x+\Delta) \sim F(x+\Delta)$.

When $T=\infty$, Theorem 4.1 reduces to part of Theorem 1 of Embrechts et al. (1979), and the other equivalent condition is $F \in \&$. Here, we give a local version of Theorem 1 of Embrechts et al. (1979).

Theorem 4.2. Let the conditions of Theorem 4.1 be valid. Furthermore, if $T<\infty$ then assume that there exists an integer $k=k(\mu)>0$ such that $\mu k^{-1}<\ln 2$ and $F_{2}^{* k^{-1}} \in \mathcal{L}_{\Delta}$. Then assertions ( $i)$ and (ii) and the following assertion are equivalent:

(iii) $F \in s_{\Delta}$.

To prove this theorem, we need the following lemma, which is a local version of Proposition 2 of Embrechts et al. (1979).

Lemma 4.1. Let $F=F_{1} * F_{2}$ be the convolution of two distributions $F_{1}$ and $F_{2}$. Assume that $F_{2} \in \mathcal{L}_{\Delta}$ or $0<F_{2}(x+\Delta) \downarrow 0$ for some $T<\infty$. Assume further that $F_{1}(x+\Delta)=$ $o(1) F_{2}(x+\Delta)$. Then $F \in s_{\Delta} \Longleftrightarrow F_{2} \in s_{\Delta}$, and either $F \in s_{\Delta}$ or $F_{2} \in s_{\Delta}$ implies that $F(x+\Delta) \sim F_{2}(x+\Delta)$.

Proof. By Proposition 3 of Asmussen et al. (2003), we easily obtain $F_{2} \in s_{\Delta} \Rightarrow F \in s_{\Delta}$. Now, we prove that $F \in s_{\Delta} \Rightarrow F_{2} \in s_{\Delta}$. By $F \in s_{\Delta}, F_{1}(x+\Delta)=o(1) F_{2}(x+\Delta)$, and Proposition 3 of Asmussen et al. (2003), we get $F_{1} * F \in s_{\Delta}$ and $F_{1} * F(x+\Delta) \sim F(x+\Delta)$. Take any $h \in \mathscr{H}_{\Delta}(F) \cap \mathscr{H}_{\Delta}\left(F_{2}\right)$. Then, by $F_{1} * F(x+\Delta) \sim F(x+\Delta)$, we have

$$
\begin{aligned}
F_{1} * F(x+\Delta) & =\left(\int_{0}^{h(x)}+\int_{h(x)}^{x}\right) F(x-y+\Delta) F_{1}(\mathrm{~d} y)+\int_{x}^{x+T} F(x-y+T) F_{1}(\mathrm{~d} y) \\
& \sim F(x+\Delta)+\int_{h(x)}^{x} F(x-y+\Delta) F_{1}(\mathrm{~d} y) ;
\end{aligned}
$$

hence

$$
\int_{h(x)}^{x} F(x-y+\Delta) F_{1}(\mathrm{~d} y)=o(1) F(x+\Delta) .
$$

If $F_{2} \in \mathcal{L}_{\Delta}$ then

$$
F(x+\Delta) \geq \int_{0}^{h(x)} F_{2}(x-y+\Delta) F_{1}(\mathrm{~d} y) \sim F_{2}(x+\Delta),
$$


giving

$$
\limsup _{x \rightarrow \infty} \frac{F_{2}(x+\Delta)}{F(x+\Delta)} \leq 1 .
$$

If $0<F_{2}(x+\Delta) \downarrow 0$, then (4.3) holds too.

Now we assume that $F_{2}(x+\Delta) \sim F(x+\Delta)$ does not hold. Then, by (4.3), there exist some $\varepsilon, 0<\varepsilon<\frac{1}{2}$, and $x_{n}$, satisfying $0<x_{n} \uparrow \infty$ as $n \rightarrow \infty$, such that

$$
\lim _{n \rightarrow \infty} \frac{F_{2}\left(x_{n}+\Delta\right)}{F\left(x_{n}+\Delta\right)} \leq 1-2 \varepsilon .
$$

By $F_{2} \in \mathcal{L}_{\Delta}\left(\right.$ or $\left.0<F_{2}(x+\Delta) \downarrow 0\right), F \in s_{\Delta}, h \in \mathscr{H}_{\Delta}\left(F_{2}\right),(4.4)$, (4.3), and integration by parts (see (2.4) and (2.5)), we know that, when $n$ is sufficiently large,

$$
\begin{aligned}
1= & \frac{F\left(x_{n}+\Delta\right)}{F\left(x_{n}+\Delta\right)} \\
= & \int_{0}^{h\left(x_{n}\right)} \frac{F_{2}\left(x_{n}-y+\Delta\right)}{F\left(x_{n}+\Delta\right)} F_{1}(\mathrm{~d} y)+\int_{h\left(x_{n}\right)}^{x_{n}-h\left(x_{n}\right)} \frac{F_{2}\left(x_{n}-y+\Delta\right)}{F\left(x_{n}+\Delta\right)} F_{1}(\mathrm{~d} y) \\
& +\int_{x_{n}-h\left(x_{n}\right)}^{x_{n}} \frac{F_{2}\left(x_{n}-y+\Delta\right)}{F\left(x_{n}+\Delta\right)} F_{1}(\mathrm{~d} y)+\int_{x_{n}}^{x_{n}+T} \frac{F_{2}\left(x_{n}-y+T\right)}{F\left(x_{n}+\Delta\right)} F_{1}(\mathrm{~d} y) \\
\leq & (1-\varepsilon) F_{1}\left(h\left(x_{n}\right)\right)+(1+\varepsilon) \int_{h\left(x_{n}\right)}^{x_{n}-h\left(x_{n}\right)} \frac{F\left(x_{n}-y+\Delta\right)}{F\left(x_{n}+\Delta\right)} F_{1}(\mathrm{~d} y)+o(1) .
\end{aligned}
$$

Letting $n \rightarrow \infty$ and using (4.2) leads to $1 \leq 1-\varepsilon$.

Therefore, it must be true that $F_{2}(x+\Delta) \sim F(x+\Delta)$ and $F_{2} \in \varsigma_{\Delta}$.

Proof of Theorem 4.2. By Proposition 3 of Asmussen et al. (2003), we find that assertion (i) implies assertion (iii). Next, we go on to prove that assertion (iii) implies assertion (i). By $\overline{F_{1}}(x)=o\left(\mathrm{e}^{-\varepsilon x}\right)$ and $G \in \mathcal{L}_{\Delta}$, we have $F_{1}(x+\Delta) \leq \overline{F_{1}}(x)=o(G(x+\Delta))$ and, from this together with (4.1), we know that $F_{1}(x+\Delta)=o\left(F_{2}(x+\Delta)\right)$. Hence, $F_{2} \in s_{\Delta}$ follows from Lemma 4.1, $F_{2} \in \mathcal{L}_{\Delta}$, and $F \in s_{\Delta}$. Then $G \in s_{\Delta}$ follows by virtue of Theorem 3.2 and $F_{2} \in s_{\Delta}$.

\section{Acknowledgements}

The authors would like to express their deep gratitude to the referee for constructive comments on the previous versions of this paper. This work was supported by the National Science Foundation of China (grant number 10271087).

\section{References}

Asmussen, S., Foss, S. And Korshunov, D. (2003). Asymptotics for sums of random variables with local subexponential behavior. J. Theoret. Prob. 16, 489-518.

Embrechts, P. And Goldie, C. M. (1982). On convolution tails. Stoch. Process. Appl. 13, 263-270.

Embrechts, P., Goldie, C. M. And Veraverbeke, N. (1979). Subexponentiality and infinite divisibility. Z. Wahrscheinlichkeitsth. 49, 335-347.

Feller, W. (1971). An Introduction to Probability Theory and Its Applications, Vol. 2, 2nd edn. John Wiley, New York. 